\title{
Interference of surface plasmon polaritions controlled by the phase of incident light
}

\author{
Xi-Feng Ren, Guo-Ping Guo*, Yun-Feng Huang, Zhi-Wei Wang, Pei Zhang and Guang-Can Guo ${ }^{1}$ \\ ${ }^{1}$ Key Laboratory of Quantum Information, University of Science and Technology of China, Hefei 230026, People's Republic of China
}

\begin{abstract}
Interference patterns of surface plasmon polaritons(SPPs) are observed in the extraordinary optical transmission through subwavelength holes in optically thick metal plate. It is found that the phase of incident light can be transferred to SPPs. We can control the destructive and constructive interference of SPPs by modulating the relative phase between two incident beams. Using a slightly displaced Mach-Zehnder interferometer, we also observe a SPPs interference pattern composed of bright and dark stripes.
\end{abstract}

PACS numbers: 78.66.Bz,73.20.MF, 71.36.+c

Surface plasmon polariton(SPP) is a surface electromagnetic wave coupling to the free electron oscillations in a metal. It can be excited when a light wave strikes a metal film under appropriate conditions. Such SPPs are involved in a wide range of phenomena[1, 2], including nanoscale optical waveguiding [3, 4, 5, 6], perfect lensing[7], extraordinary optical transmission[8], subwavelength lithography[9] and ultrahigh-sensitivity biosensing[10]. It has also been experimentally proved that SPPs are also useful in the investigation of quantum information[11, 12, 13]. Since surface plasmon-based photonics(plasmonics) has both the capacity of photonics and the miniaturization of electronics, it may offer us a solution to the size-compatibility problem[2]. To realize the full potential technology of palsmonics, we need to construct a general frame work to describe the propagating, diffraction and interference of SPPs.

Interference of SPPs is first studied by the group of Lezec[14]. They have shown that light transmission through a slit milled in an Ag film can be passively enhanced(suppressed) as a result of constructive(destructive) interference with a SPP launched by a groove nearby. Efficient unidirectional nanoslit couplers for SPPs[15] and alloptical modulation by plasmonic excitation of CdSe quantum dots [16] are also realized based on the interference of SPPs. A double-slit experiment with SPPs is presented which reveals the analogue between SPPs propagating along the surface of metallic structures and light propagating in conventional dielectric components[17]. It is also proved that SPPs can be excited with a focused laser beam at normal incidence to a metal film without any protrusions and holes, while the intensity distribution on the metal surface is partly dominated by interference between counterpropagating plasmons[18]. In these works, the construction or destruction of interference pattern is determined by the propagating distance of SPPs on the metal surface. For example, in [16], the transmitted energy is varied with the distance between the slit and the groove. Of course, many samples are needed to give a full characterization of the interference pattern.

It is important for us to control the interference pattern of SPPs on a given sample in the approach of the chip-based

*gpguo@ustc.edu.cn plasmonics. One way is to change the wavelength of incident light, as in [3], where the transmission of the plasmonic waveguide-ring resonator is varied with the light wavelength. However, this method of varying wavelength may be not feasible in the practical application. In this paper, we show that the phase of the incident light can be transferred to the SPPs, which provides us a potential method to modulate the interference pattern of SPPs using the incident lights. We use two light beams to excite SPPs. By controlling the relative phase of two beams, we can observe the interference pattern of SPPs for serval periods. The transmission can be tuned continuously from minimum to maximum with a ratio about 7 , even when the power of the incident light is kept stable. To give an intuitional illustration, we also take several pictures of the interference pattern composed of bright and dark stripes using a charge coupled device(CCD) camera behind a slightly displaced Mach-Zehnder(MZ) interferometer. The present method may be valuable in the future application of plasmonics due to the well-established technology on the linear optical elements.

The metal plate used in our experiment to excite SPPs is produced as follows: after subsequently evaporating a 3- $\mathrm{nm}$ titanium bonding layer and a 135- $\mathrm{nm}$ gold layer onto a $0.5-\mathrm{mm}$ thick silica glass substrate, a Electron Beam Lithography System (EBL, Raith 150 of Raith Co.) is used to produce cylindrical holes $(200 \mathrm{~nm}$ diameter) arranged as a square lattice (600 $\mathrm{nm}$ period). The area of the hole array is $300 \mu \mathrm{m} \times 300 \mu \mathrm{m}$.

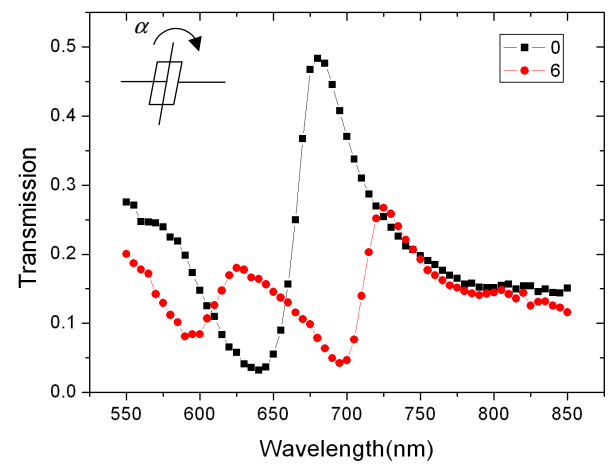

FIG. 1: (Color online)Hole array transmittance as a function of wavelength for vertical polarized light with tilt angle $\alpha=0^{\circ}$ (blue square dots) and $6^{\circ}$ (red round dots). Inset is an illustration of tilt direction. 
Transmission spectra of the hole array are recorded by a Silicon avalanche photodiode (APD) single photon detector coupled with a monochromator through a fiber. White light from a stabilized tungsten-halogen source passes through a single mode fiber and a polarizer (only vertical polarized light can pass), then illuminates on the sample. The hole array is set between two lenses with the focus of $35 \mathrm{~mm}$, so that the light is normally incident on the hole array with a cross sectional diameter about $20 \mu \mathrm{m}$ and covers hundreds of holes. The light exiting from the hole array is launched into the monochromator. The transmission spectra are shown in Fig. 1. The black square dots are measured with tilt angle $\alpha=0^{\circ}$ and red round dots with $\alpha=6^{\circ}$. The reason for tilting the metal plate will be explained below.

To control the interference pattern of SPPs by the incident lights, firstly we need to prove that the phase of excited SPPs is correlated with that of incident light. This is verified by using a collinear polarization MZ interferometer as shown in Fig. 2. The advantage of this kind of MZ interferometer is the stability against the environment. We consider the case when the metal plate is removed from the twin-lenses at first. White light from a stabilized tungsten-halogen source passes through single mode fiber and $4 \mathrm{~nm}$ filter (center wavelength $702 \mathrm{~nm}$ ) to provide photons at the wavelength of $702 \mathrm{~nm}$. Only vertical polarized photons can transmit the first polarization beam splitter(PBS). When the first half wave plate(HWP) is rotated to $-22.5^{\circ}$, the photons in the state $|V\rangle$ will be changed into the state $(|H\rangle+|V\rangle) / \sqrt{2}$. Then these photons go through the birefringent crystal(BC), where they get a phase difference $\Delta \varphi$ between horizontal and vertical polarization modes. The state is thus in the form of $\left(|H\rangle+e^{i \Delta \varphi}|V\rangle\right) / \sqrt{2}$. After the second $\operatorname{HWP}\left(+22.5^{\circ}\right)$, the state is transformed into $1 / 2\left(\left(1+e^{i \Delta \varphi}\right)|H\rangle+\left(1-e^{i \Delta \varphi}\right)|V\rangle\right)$. Then the photons are separated by the second PBS which also only permits the transmission of $V$ polarized photons. Since there is a phase difference between the two parts of transmitted $V$ polarized photons, the counts can be varied with this phase difference due to the interference effect. The experiment results are shown in Fig. 3a (Black square dots), which fits the theoretical interference pattern nicely in the $|V\rangle$ basis

$$
R_{V}=(\sin (\Delta \varphi / 2))^{2} .
$$

Now we put the metal plate with hole array between the twin lenses. The light irradiates the metal plate normally and the transmission efficiency is measured. In this case, photons are first transformed into SPPs and then back to photons[8]. Fig. $3 \mathrm{a}$ (Red round dots) are the experimental results, which also fits the theoretical calculation nicely. This gives the evidence that the phase of the input light can be transferred to the SPPs.

However, this curve may result from the interference of the transmitted photons on the second PBS, not the SPPs on the metal surface. It is necessary for us to do a further investigation. We remove the second PBS from the setup to avoid this probability. It is observed that the counts are kept constant and there is no interference phenomenon as shown in Fig. $3 b$ when the metal plate is moved away. The reason is that all the photons are detected in this case. Then the metal plate is placed between the two lenses at the tilted angle of $\alpha=6^{\circ}$. In this case, due to the removal of SPPs degeneracy, the transmission spectra is changed for the vertically polarized photons $[8,19,20]$. The transmission peak at $680 \mathrm{~nm}$ is divided into two peaks at the wavelength of $625 \mathrm{~nm}$ and $725 \mathrm{~nm}$ respectively. It is noted that the transmission spectra for horizontal polarized photons is not influenced. The transmission efficiency of $702 \mathrm{~nm}$ light with vertical polarization is only $0.051 \%$ at the tilted angle of $6^{\circ}$, which comes from the direct transmission from the nano-scale holes. While for horizontal polarized light, the transmission is about $0.354 \%$ due to the SPPs assisted transmission process [8]. Thus most of the counts are coming from the horizontal polarized photons. A clear interference pattern is observed in this situation as shown in Fig. 3c. This phenomenon must come from the interference of SPPs excited on the metal surface by the horizontal polarized light. Of course, the pattern is contrary to the previous cases as shown in Fig. 3a. The ratio between maximum transmission and minimum transmission is about 7, corresponding to the ratio of transmission efficiency between the horizontal and vertical polarized lights, which is determined by titled angle $\alpha$ of the metal plate. So we can draw the conclusion that the interference of SPPs can be expediently controlled by the phase of illuminated light with linear optical elements.

We also get an intuitional illustration of the interference of

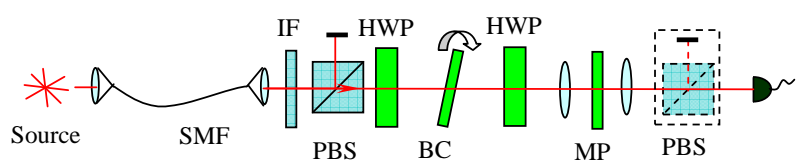

FIG. 2: (Color online)Experimental setup to observe the interference pattern of SPPs for $702 \mathrm{~nm}$ wavelength light. The collinear polarization MZ interferometer is composed of two PBS, two HWPs and a birefringent crystal. The birefringent crystal is tilted to generate a phase difference $\Delta \varphi$ between horizontal and vertical polarization modes. The metal plate is set between two lenses with the focus of $35 \mathrm{~mm}$ and the second PBS is removed in some cases.

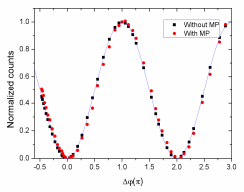

(a)

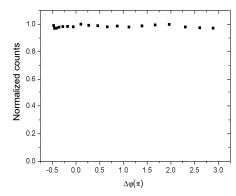

(b)

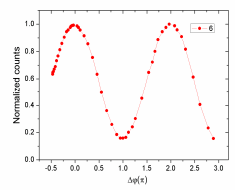

(c)
FIG. 3: (Color online)Interference pattern for light with $702 \mathrm{~nm}$ wavelength. (a) The second PBS is placed in the experimental setup. Black square dots are normalized counts without the metal plate, while red round dots with the metal plate. They both fit the theoretical calculation(the line) well, which sustain the conclusion that the phase of the illuminating light can be transferred to the SPPs. (b) The counts are kept constant when we take the second PBS out and the metal plate away. (c) An interference pattern is observed when the metal plate is placed between the two lenses at the tilted angle of $6^{\circ}$, even without the second PBS. The interference of SPPs can be fully controlled by the phase difference $\Delta \varphi$. 
SPPs. A path MZ interferometer is used as shown in Fig. 4. The essence of this MZ interferometer is equal to the previous collinear polarization interferometer, while the phase $\Delta \varphi$ comes from the path difference. In this interferometer, the position of two light beams can be tuned slightly by the reflector(R)(see Fig. 4). The collinear of the two light beams after the beam splitter(BS) is slightly destroyed in the horizontal direction by displacing the reflector. An interference pattern with bright and dark stripes in the vertical direction is recorded by a CCD when the second PBS is placed in the setup(Fig. 5a). If we take the second PBS away, there is no interference pattern(Fig. 5b). When we put the metal plate in at the tilted angle of $6^{\circ}$, a similar image of bright and dark stripes appears as shown in Fig. 5c, which is a vivid picture of interference of SPPs. The three pictures correspond to the cases of Fig. 3a(Black square dots), Fig. 3b and Fig. 3c respectively. Since the wavelength of incident light is $632.8 \mathrm{~nm}$, the SPPs are excited by the vertical polarized light, which is different from the case of $702 \mathrm{~nm}$.

In conclusion, interference of SPPs is observed by controlling the phase of incident light. Due to the well-established technology on the linear optical elements, we can modulate the interference pattern of SPPs from destructive to constructive continuously. Our method may be useful in the future investigation of plasmonics.

This work was funded by the National Fundamental Research Program(No. 2006CB921900), National Natural Science Foundation of China (No. 10604052), Chinese Academy

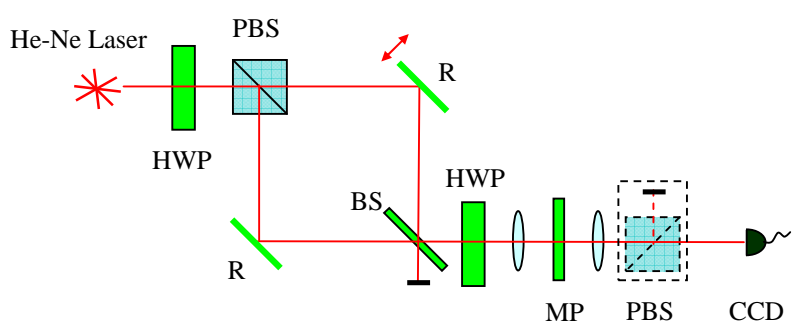

FIG. 4: (Color online)Experimental setup to observe the interference pattern of SPPs for $632.8 \mathrm{~nm}$ wavelength light. The path difference of $\mathrm{MZ}$ interferometer gives the phase difference $\Delta \varphi$. A reflector is displaced slightly to observe the interference pattern of bright and dark stripes.

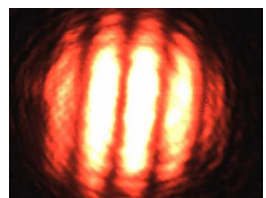

(a)

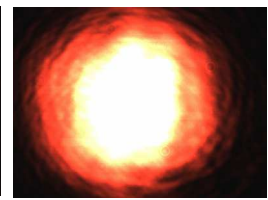

(b)

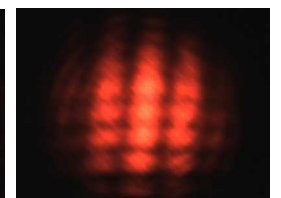

(c)
FIG. 5: (Color online)Energy distribution recorded by the CCD. (a) General interference pattern of a displaced MZ interferometer with the second PBS.(b) Energy distribution when the second PBS is removed. The metal plate is moved out for (a) and (b). (c) Interference pattern of SPPs is observed even when the second PBS is removed. The metal plate is tilted $6^{\circ}$ to excite SPPs. of Sciences International Partnership Project, the Program of the Education Department of Anhui Province (Grant No.2006kj074A). Xi-Feng Ren also thanks for the China Postdoctoral Science Foundation (20060400205) and the K. C. Wong Education Foundation, Hong Kong.

[1] W. L. Barnes, A. Dereux, T. W. Ebbesen, Nature 424, 824 (2003).

[2] E. Ozaby, Science 311, 189 (2006).

[3] S. I. Bozhevolnyi, V. S. Volkov, E. Devaux, J. Y. Laluet, T. W. Ebbesen, Nature 440, 508 (2006).

[4] J. Takahara, S. Yamagishi, H. Taki, A. Morimoto, T. Kobayashi, Opt. Lett. 22, 475 (1997).

[5] J. Takahara, T. Kobayashi, Opt. Photonics News 15, 54 (2004).

[6] R. Zia, J. A. Schuller, A. Chandran, M. L. Brongersma, Mater. Today 9, 20 (July/August 2006).

[7] J. B.Pendry, Phys. Rev. Lett. 85, 3966 (2000).

[8] T.W. Ebbesen, H. J. Lezec, H. F. Ghaemi, T. Thio, and P. A. Wolff, Nature 391, 667 (1998).

[9] N. Fang, H. Lee, C. Sun, X. Zhang, Science 308, 534 (2005).

[10] B. Liedberg, C. Nylander, I. Lundstrom, Sens. Actuators 4, 299 (1983).

[11] E. Altewischer, M. P. van Exter and J. P. Woerdman, Nature 418, 304 (2002).

[12] S. Fasel, F. Robin, E. Moreno, D. Erni, N. Gisin and H. Zbinden, Phys. Rev. Lett. 94, 110501 (2005).

[13] X. F. Ren, G. P. Guo, Y. F. Huang, C. F. Li, and G. C. Guo, Europhys. Lett. 76, 753 (2006).

[14] G. Gay, O. Alloschery, B. Viaris de Lesegno, C. O’D wyer, J. Weiner and H. J. Lezec, Nature Phys. 2, 262 (2006).

[15] F. Lopez-Tejeira, S. G. Rodrigo, L. Martin-Moreno, F. J. Garcia-Vidal, E. Devaux, T. W. Ebbesen, J. R. Krenn, I. P. Radko, S. I. Bozhevolnyi, M. U. Gonzalez, J. C. Weeber and A. Dereux, Nature Phys. 3, 324 (2007).

[16] D. Pacifici, H. J. Lezec, and H. A. Atwater, Nature Photonics 1, 402 (2007).

[17] R. Zia, and M. L. Brongersma, Nature Nanotechnology 2, 426 (2007).

[18] A. Bouhelier, F. Ignatovich, A. Bruyant, C. Huang, G. Colas des Francs, J. C. Weeber, A. Dereux, G. P. Wiederrecht, and L. Novotny, Opt. Express 32, 2535 (2007).

[19] C. Genet, M. P. van Exter, and J. P. Woerdman, J. Opt. Soc. Am. A. 22, 998 (2005).

[20] X. F. Ren, G. P. Guo, P. Zhang, Y. F. Huang, Z. W. Wang, and G. C. Guo, arXiv:physics/0703230, to be published in Appl. Phys. B. 